\title{
Lockdown contra a Covid-19 funciona ou não? Um estudo de caso com o município de Araraquara
}

\author{
Marilaine Colnago ${ }^{1}$ \\ UNESP, Campus de Rosana, Rosana, SP \\ Wallace Casaca ${ }^{2}$ \\ UNESP, Campus de Rosana, Rosana, SP \\ Cássio Machiaveli Oishi ${ }^{3}$ \\ UNESP, Faculdade de Ciências e Tecnologias, Presidente Prudente, SP \\ Fábio Vinicius Amaral ${ }^{4}$ \\ UNESP, Faculdade de Ciências e Tecnologias, Presidente Prudente, SP \\ José Alberto Cuminato 5 \\ USP, Instituto de Ciências Matemáticas e de Computação, São Carlos, SP
}

\begin{abstract}
Resumo. O presente trabalho apresenta uma análise da evolução da Covid-19 no município de Araraquara, localizado no interior do Estado de São Paulo. Essa análise tem como objetivo investigar a eficiência do lockdown como estratégia de contenção no avanço do novo coronavírus, através da análise dos dados de casos confirmados, internações, óbitos e do número efetivo de reprodução do vírus. Para isto, foi realizado um estudo comparativo envolvendo os dados do município de São Carlos-SP, bem como o número de novos óbitos por 100 mil habitantes dos municípios paulistas de Presidente Prudente, Dracena e Jaú que, assim como São Carlos, não implementaram um regime de lockdown permanente. Os resultados obtidos demonstram que a adoção de medidas mais rígidas de redução de circulação de pessoas foi capaz de diminuir significativamente os indicadores relativos à disseminação da Covid-19 em Araraquara. A partir da análise dos dados, foi também possível constatar que, no mesmo período em que os índices da Covid-19 de Araraquara estavam em queda, o oposto ocorreu em São Carlos, que apresentou alta nos índices. Além disso, em relação à curva de novos óbitos ponderados por população, houve redução em Araraquara após o término do lockdown, e crescimento nos demais municípios analisados.
\end{abstract}

Palavras-chave. Covid-19, Análise de Dados, Lockdown, Modelos Matemáticos.

\section{Introdução}

A Covid-19, doença causada pelo coronavírus denominado SARS-CoV-2, foi identificada pela primeira vez na China, em dezembro de 2019. Em 30 de janeiro de 2020, a Organização Mundial da Saúde (OMS) declarou que a epidemia da Covid-19 constituía uma Emergência de Saúde Pública de Importância Internacional e, em 11 de março de 2020, uma pandemia em escala global.

De acordo com [6], além da queda de dois Ministros da Saúde em menos de um mês, o Brasil contou com a falta de articulação entre os governos federal, estadual e municipal, e com a disseminação de notícias falsas acerca de tratamentos contra a Covid-19. Esses fatores, juntamente

\footnotetext{
${ }^{1}$ marilaine.colnago@unesp.br

2 wallace.casaca@unesp.br

3 cassio.oishi@unesp.br

${ }^{4}$ fabio.amaral@unesp.br

5 jacumina@icmc.usp.br
} 
com a grande desigualdade social existente no país, e a rápida propagação do vírus, vem causando uma sequência de recrudescimentos ("ondas") da pandemia, resultando na falta de oxigênio e de leitos nos hospitais. Parte desses efeitos foram também observados no Estado de São Paulo que, frente às altas taxas de ocupação hospitalar, buscou priorizar intervenções não-farmacêuticas (NPI - Non-Pharmaceutical Interventions) como medidas mais rígidas de enfrentamento à Covid-19 a fim de tentar dirimir a transmissão do vírus.

Segundo [2], estratégias para controlar a Covid-19 com sucesso dependerão tanto de um bom programa de vacinação, quanto de um conjunto efetivo de NPIs, com destaque para o distanciamento social, testagem massiva e isolamento dos indivíduos contaminados. Essas medidas, adotadas de maneira mais incisiva, são necessárias para garantir o não colapso do sistema de saúde [5]. De fato, em [4], os autores analisaram quatro tipos de intervenções não-farmacêuticas, mostrando que todas reduzem a transmissão do vírus e são mais efetivas quando implementadas de forma concomitante. Somente com a quarentena, houve redução de 10,6\% no índice de transmissibilidade do vírus. Já com a quarentena combinada com a restrição do transporte público, houve queda de $17,83 \%$ e, se aliada ao distanciamento social, $38,58 \%$. Se considerarmos as três NPIs juntas, esse valor chegou a $54,12 \%$ e, se combinadas à utilização de máscaras, $62,81 \%$ de redução.

Mesmo com as restrições de circulação impostas pelo Plano São Paulo ${ }^{6}$, as NPIs adotadas no âmbito estadual não foram suficientes para conter o avanço do vírus em algumas regiões do estado. Dessa maneira, medidas mais restritivas começaram a ser debatidas e adotadas por parte de algumas prefeituras municipais de São Paulo, como foi o caso do município de Araraquara que, ao registrar um recorde de novos casos diários e ver seu sistema de saúde colapsar em questão de semanas, decretou lockdown por 10 dias ininterruptos, entre 21 de fevereiro à 2 de março de 2021 .

Diante dos recorrentes questionamentos acerca do grau de efetividade de medidas restritivas mais austeras como o lockdown rígido (não-intermitente), cuja discussão fomenta o debate entre sociedade civil, cientistas, e diferentes segmentos do poder público, o presente estudo propõe uma análise detalhada dos dados epidemiológicos da Covid-19 em Araraquara, comparando-os com os índices métricos da pandemia de outros municípios paulistas que optaram pela não implementação de um regime de lockdown permanente e rígido.

\section{Metodologia}

\subsection{Dados Observados e Critérios de Seleção}

Os dados do município de Araraquara foram escolhidos para o presente estudo, em razão da cidade ser a primeira com mais de 100 mil habitantes a ter decretado um estado de lockdown contínuo, suspendendo a circulação de veículos e de pessoas ao longo do dia, a não ser em casos excepcionais, e todos os tipos de serviços que não tinham relação direta com a área da saúde, incluindo transporte público e supermercados, que só funcionaram pelo sistema de delivery.

Para conduzir análises comparativas com os dados de Araraquara, optou-se pelo município de São Carlos, que dista menos de $50 \mathrm{~km}$ da mesma. Além de serem cidades geograficamente próximas, ambas possuem várias semelhanças em termos de índices demográficos e socioeconômicos, conforme destacado na Tabela $1^{7}$. Ademais, até meados de janeiro-2021, um mês antes de Araraquara ter notificado publicamente seus primeiros casos da variante P.1, os dois municípios apresentavam curvas epidemiológicas similares, conforme evidencia a Figura 1, que destaca a média móvel dos novos casos confirmados nos dois municípios no período de abril-2020 até meados de janeiro-2021. Apesar de apresentarem diferenças de amplitude em determinados subperíodos como, entre agosto

\footnotetext{
${ }^{6}$ Disponível em: https://www.saopaulo.sp.gov.br/planosp. Acesso em 22 de abril de 2021.

${ }^{7}$ Dados extraídos da Fundação SEADE (Sistema Estadual de Análise de Dados). Disponível em: https://perfil.seade.gov.br/. Acesso em: 24 de março de 2021.
} 
e novembro-2020, a "assinatura" de tendência de ambas às curvas foi similar, sobretudo, a partir de novembro até meados de janeiro-2021, data em que as ações de enfrentamento para conter o avanço do vírus nos dois municípios começaram a se distinguir significativamente, especialmente após 21 de fevereiro de 2021, com a adoção do regime de lockdown rígido em Araraquara.

Tabela 1: Dados demográficos e socioeconômicos dos municípios de Araraquara e São Carlos.

\begin{tabular}{lcc}
\hline & Araraquara & São Carlos \\
\hline População & 228.793 & 244.036 \\
Área territorial $\left(\mathrm{km}^{2}\right)$ & $1.003,63$ & $1.136,91$ \\
Densidade demográfica (habitantes $\left./ \mathrm{km}^{2}\right)$ & 227,96 & 214,65 \\
Analfabetismo (15 anos ou mais) & $3,62 \%$ & $3,66 \%$ \\
Ensino médio completo (18 a 24 anos) & $70,15 \%$ & $67,63 \%$ \\
Renda per capita (R\$) & 891,74 & 923,63 \\
IDH & 0,815 & 0,805 \\
Participação no PIB do Estado & $0,4256 \%$ & $0,5054 \%$ \\
\hline
\end{tabular}

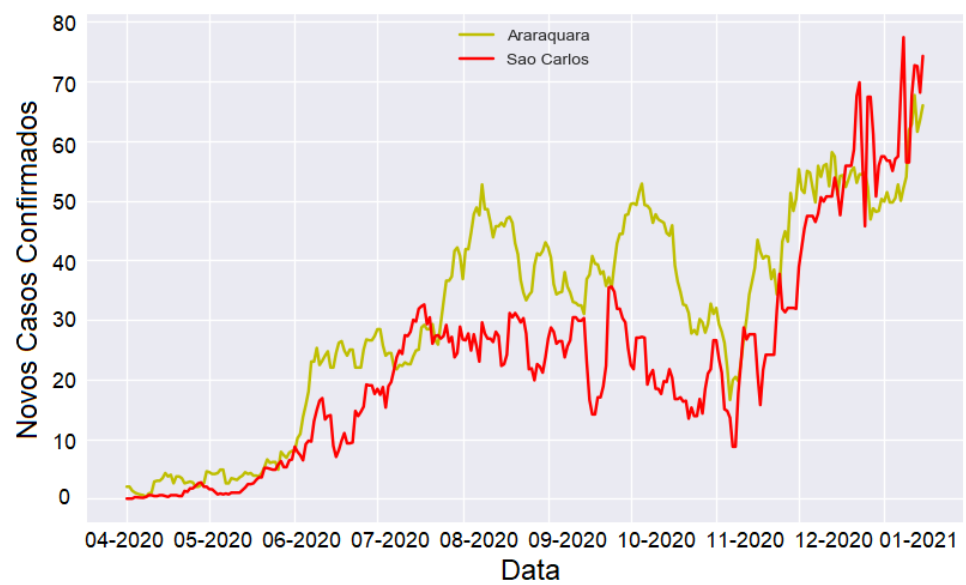

Figura 1: Média móvel dos novos casos confirmados ente 01 de abril de 2020 e 16 de janeiro de 2021.

Em adição à implementação unilateral do lockdown por parte de Araraquara, é importante também pontuar que, embora ambos os municípios integrem o mesmo Departamento Regional de Saúde do Estado de SP no âmbito do chamado Plano São Paulo, que regulamenta a implementação de políticas públicas de enfrentamento da Covid-19 por região do estado, São Carlos se restringiu em apenas seguir as recomendações do referido Plano, portanto não adotando nenhuma outra medida adicional mais restritiva que possa se assemelhar ao lockdown. A decisão pela não implementação de um regime de lockdown contínuo não se limitou à apenas o município de São Carlos, já que cidades como Jaú, Presidente Prudente e Dracena, que apresentaram um aumento significativo no número de casos confirmados e óbitos no primeiro trimestre de 2021, também se contiveram à seguir as recomendações do Plano São Paulo. Por essa razão, além da análise das curvas epidemiológicas de São Carlos e Araraquara, será apresentada neste estudo uma investigação dos novos óbitos por cada 100 mil habitantes dos municípios supracitados anteriormente. 


\subsection{Base de Dados, Variáveis Epidemiológicas e Modelos Matemáticos}

A base de dados utilizada no presente estudo foi extraída da plataforma SP Covid-19 Info Tracker $^{8}$, que coleciona dados recolhidos diretamente dos boletins epidemiológicos das prefeituras. Além dos dados brutos disponibilizados pelos municípios, a plataforma também efetua o computo de índices estatísticos e epidemiológicos como taxas de variação, letalidade, mortalidade, número de casos por 100 mil habitantes (incidência), casos confirmados ativos, entre outros.

As curvas de casos ativos, que serão exploradas no presente trabalho, foram determinadas a partir da metodologia matemática proposta em [7], que relaciona as variáveis $I(t)$, o número acumulado de casos (Infections), $D(t)$, o total acumulado de óbitos (Deaths), a variável Rec $(t)$, que corresponde aos casos de recuperação (Recoveries), e finalmente, o tempo de incubação do vírus e o tempo médio para a recuperação plena de um indivíduo. Em alinhamento com [7], foi considerado como caso resolvido, que é quando um indivíduo está plenamente recuperado da Covid-19, os casos que não resultaram em óbito e que já decorreram 21 dias a partir da data de início dos sintomas, visto que o tempo médio de recuperação total de um indivíduo é de 20.8 dias, conforme detalha [3]. A partir dessa simplificação, o número de casos confirmados ativos $A(t)$ pode ser então estimado a partir da seguinte expressão matemática:

$$
A(t)=I(t)-D(t)-\operatorname{Rec}(t), \operatorname{com} \operatorname{Rec}(t)=I(t-21)-D(t-21) .
$$

Outra métrica importante neste contexto é o número efetivo de reprodução do novo coronavírus, também conhecido como "taxa" de propagação/transmissão do vírus. Em suma, esse índice epidemiológico, simbolizado por $R_{t}$ ou $R t$, determina o número médio de pessoas que são infectadas por um único indivíduo com o vírus ativo, dado uma população de indivíduos suscetíveis. De maneira simplificada, um valor de $R t$ igual a 1 significa que cada pessoa contaminada transmite o vírus para exatamente uma nova pessoa suscetível. Caso o valor esteja acima de 1, cada infecção existente levará à mais de uma nova infecção. Nesse caso, a doença será transmitida rapidamente entre as pessoas, podendo causar um surto ou epidemia, indicando a necessidade de adoção de medidas mais austeras. Finalmente, se o Rt estiver abaixo de 1, o número de novas infecções será menor do que o total de infectados ativos da geração anterior, portanto reduzindo o total de infectados.

Para estimar o índice $R t$ do novo coronavírus, utilizou-se a metodologia apresentada em [1]. Tal abordagem combina o uso de três mecanismos, a saber: (i) o modelo matemático de epidemiologia SIRD (Susceptible-Infected-Recovered-Deceased), (ii) técnicas de inteligência artificial (redes neurais e aprendizado de máquina), para fins de captura de padrões das curvas epidemiológicas das populações analisadas, e (iii) dados epidemiológicos atualizados, adquiridos através dos boletins epidemiológicos das prefeituras municipais do Estado de SP.

\section{Resultados e Discussão}

A seguir, são apresentadas as análises comparativas ao longo de 17 de janeiro à 10 de abril de 2021 (semanas epidemiológicas 3 à 14). Em cada caso, foi calculada a média móvel de 7 dias. As faixas azuis nos gráficos destacam o período de lockdown implementado por Araraquara.

A Figura 2(a) apresenta o número de novos casos confirmados em cada município. O pico no município de Araraquara ocorre próximo ao início do período de lockdown, mais especificamente entre os dias 15 e 26 de fevereiro. Nota-se que, após esse período, o número de novos casos sofre uma redução significativa e, após algumas semanas, passa a se manter abaixo dos valores registrados por São Carlos que, por sua vez, passa a apresentar valores tão elevados quantos os de Araraquara durante seu pico. Na Figura 2(b), são plotadas as comparações dos casos ativos. Assim como no cenário anterior, Araraquara apresenta um pico que decresce rapidamente após o término da fase

\footnotetext{
${ }^{8}$ Disponível em: www.spcovid.net.br. Acesso em: 20 de abril de 2021.
} 
de lockdown. Por outro lado, a curva de São Carlos ascende próximo a 18 de março, mantendo-se inclusive acima da curva de Araraquara nas semanas finais. Já a Figura 2(c) traz os novos óbitos confirmados e, apesar de São Carlos não apresentar um pico tão acentuado como Araraquara, também não apresenta nenhuma queda expressiva ao longo do período. No período pós-lockdown, em que se observa uma rápida redução nos óbitos em Araraquara, o oposto não ocorre em São Carlos: na verdade, este passa a apresentar mais óbitos do que Araraquara nas semanas finais.

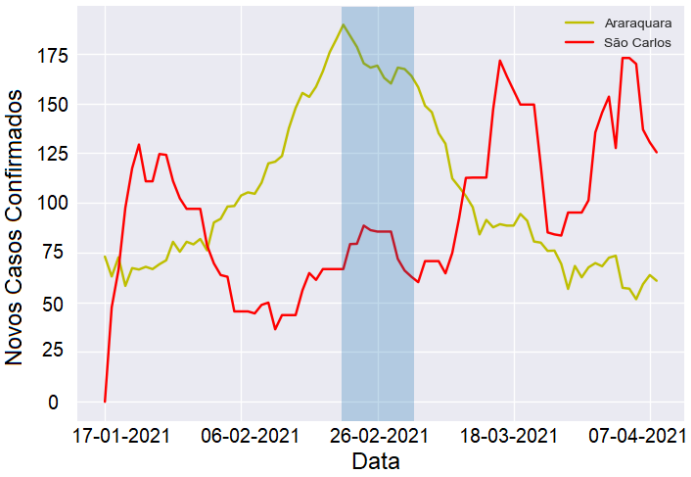

(a)

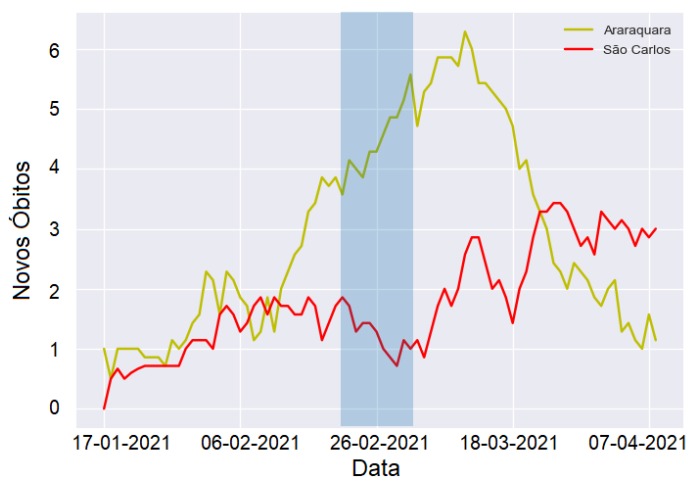

(c)

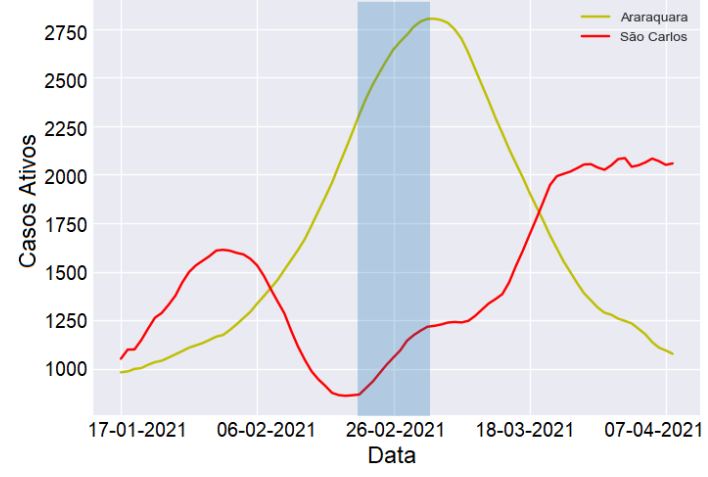

(b)

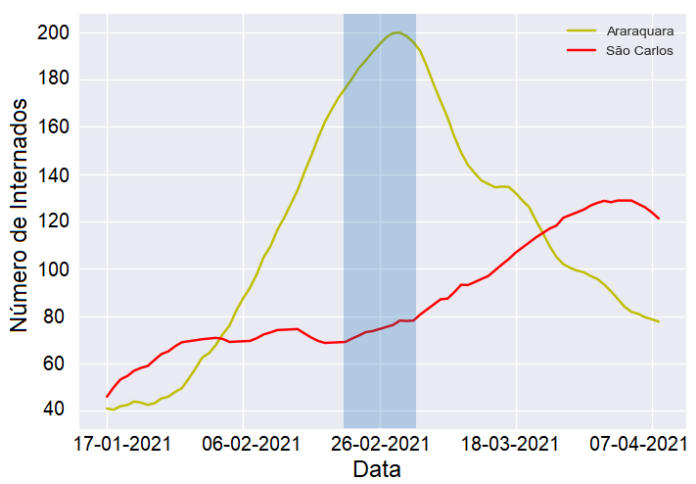

(d)

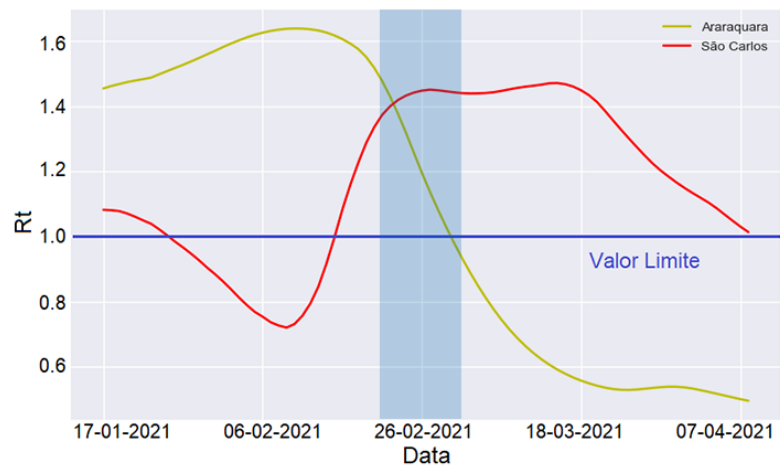

(e)

Figura 2: (a) Novos casos, (b) casos ativos, (c) novos óbitos, (d) internados e (e) número de reprodução $R t$ ("taxa" de transmissão) em Araraquara e São Carlos. 
A Figura 2(d) traz o total de pessoas internadas, por dia, e assim como nos demais casos, há uma queda acentuada na curva de Araraquara após o lockdown ante à ascensão das hospitalizações em São Carlos. Por fim, a Figura 2(e) apresenta o índice $R t$ de cada um dos municípios. É possível notar que, entre 15 de fevereiro e 18 de março, a curva $R t$ de Araraquara decresce rapidamente, passando a se estabilizar, abaixo de 0,6 , nas semanas seguintes. Considerando a curva $R t$ de São Carlos, esta inicialmente apresenta valores superiores à 0,7 , elevando-se e mantendo-se acima de 1,0 aproximadamente a partir de 15 de fevereiro, embora com tendência de redução.

Portanto, a análise qualitativa dos dados evidencia que, após o período de lockdown, a situação pandêmica em Araraquara melhora significativamente, tal como apontado pela redução dos cinco índices avaliados. Por outro lado, São Carlos apresenta a situação oposta, conforme analisado.

Na Figura 3, uma análise comparativa dos novos óbitos por 100 mil habitantes é apresentada, a qual envolve, além de Araraquara e São Carlos, os dados de Dracena, Jaú e Presidente Prudente: municípios do interior paulista que também registraram grande incidência da variante P.1. Dentre os municípios analisados, destaca-se Dracena que, ao contrário de Araraquara, não adotou um regime de lockdown permanente. Com relação à Jaú, observa-se que a maior ocorrência de novos óbitos foi registrada em um intervalo de mais de um mês (25 janeiro e 10 de março), coincidindo com o período em que a Santa Casa do município anunciou um colapso em seu sistema de saúde, ficando sem leitos de UTI Covid disponíveis ${ }^{9}$. Além disso, no período mencionado, Jaú atinge um número de óbitos por habitante maior do que Araraquara.

Com relação à Presidente Prudente e São Carlos, estas apresentam curvas que estão inicialmente pareadas com Araraquara, entretanto, em meados de fevereiro, há uma ascensão nos óbitos de Araraquara, chegando ao seu pico próximo ao dia 8 de março. Após uma semana, observa-se o efeito do lockdown em Araraquara na contenção da variante P.1, a partir do rápido decrescimento de seus óbitos e do menor índice registrado dentre todas as cinco cidades avaliadas nos últimos dias. Já em São Carlos e Presidente Prudente, o número de óbitos se eleva e se mantém em crescimento a partir de 26 de fevereiro. Finalmente, cabe destacar que, dentre os municípios analisados, Araraquara foi o único que não apresentou aumento de novos óbitos após o declínio.

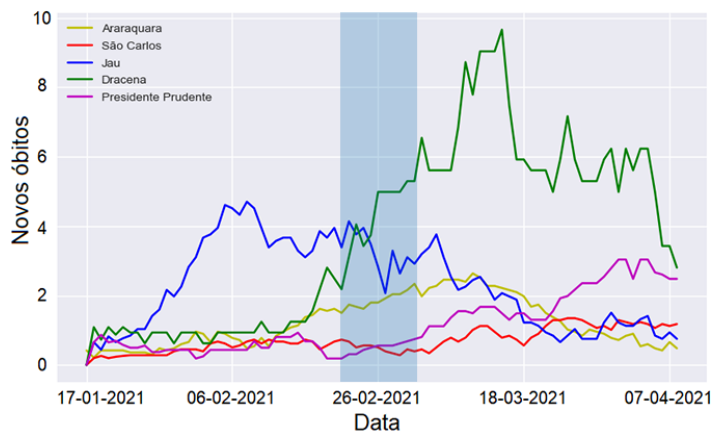

\begin{tabular}{ccccc}
\hline \multirow{2}{*}{ Cidade } & & $\mathbf{P}_{\mathbf{1}}$ & $\mathbf{P}_{\mathbf{2}}$ & $\mathbf{P}_{\mathbf{3}}$ \\
& & $17 / 01-13 / 02$ & $14 / 02-13 / 03$ & $14 / 03-10 / 04$ \\
\hline \multirow{2}{*}{ Araraquara } & Óbitos & 18,88 & 56,66 & 27,28 \\
& Variação & - & $200 \%$ & $-52 \%$ \\
\hline \multirow{2}{*}{ Dracena } & Óbitos & 26,18 & 154,86 & 133,06 \\
& Variação & - & $492 \%$ & $-14 \%$ \\
\hline \multirow{2}{*}{ Jaú } & Óbitos & 79,02 & 84,28 & 28,32 \\
& Variação & - & $7 \%$ & $-66 \%$ \\
\hline \multirow{2}{*}{ Presidente } & Óbitos & 15,20 & 24,74 & 65,12 \\
Prudente & Variação & - & $63 \%$ & $163 \%$ \\
\hline \multirow{2}{*}{ São Carlos } & Óbitos & 13,36 & 18,88 & 30,26 \\
& Variação & - & $41 \%$ & $60 \%$ \\
\hline
\end{tabular}

Figura 3: (Esquerda) Óbitos, por 100 mil habitantes, e (Direita) total de óbitos, por 100 mil habitantes, em cada período $\mathbf{P}_{\mathbf{i}}$ de 28 dias (4 semanas epidemiológicas) e variação percentual entre períodos consecutivos.

Finalmente, a Tabela da Figura 3 sumariza o total de óbitos, por 100 mil habitantes, em cada período $\mathbf{P}_{\mathbf{i}}$ de quatro semanas epidemiológicas (28 dias). Ao longo de $\mathbf{P}_{\mathbf{1}}$, destaca-se Jaú, enquanto que no período $\mathbf{P}_{\mathbf{2}}$, tem-se Dracena, Jaú e Araraquara atingindo os maiores números de óbitos. Após o término do lockdown, implementado num período de 10 dias no "mês" epidemiológico $\mathbf{P}_{\mathbf{2}}$, Araraquara registra uma redução de $52 \%$ nos novos óbitos entre $\mathbf{P}_{\mathbf{2}}$ e $\mathbf{P}_{\mathbf{3}}$, segunda maior redução

\footnotetext{
${ }^{9}$ Notícia reportada em: www.g1.globo.com/sp/bauru-marilia/noticia/2021/01/26/sem-vagas-de-uti-santa-casade-jau-pede-ajuda-para-transferir-pacientes-estamos-em-colapso.ghtml. Acesso em: 20 de abril de 2021.
} 
registrada, estando apenas atrás de Jaú. No entanto, é importante pontuar que Jaú mantém um número mais elevado de óbitos do que Araraquara em todos os subintervalos. Finalmente, nos casos de Presidente Prudente e São Carlos, houve aumento em todos os períodos.

\section{Conclusões}

A partir da análise conduzida no estudo, conclui-se que o lockdown, objetivando-se assegurar o cumprimento do distanciamento social, foi efetivo para a redução de novos casos, internações e óbitos causados pela Covid-19 em Araraquara, sobretudo, em um cenário de alta incidência da variante P.1. Essa afirmação foi verificada a partir das comparações realizadas entre o referido município e seu vizinho, São Carlos, em vista deles seguirem em direções opostas após o período de lockdown: os números em Araraquara sofreram uma queda significativa, enquanto os mesmos índices em São Carlos se mostraram em crescimento/estagnação. O mesmo foi observado com os óbitos de outros municípios que não adotaram um regime de lockdown contínuo.

Dessa forma, conclui-se que a adoção de medidas mais austeras como o lockdown, impacta diretamente na redução de novas contaminações e mortes ocasionadas pelo SARS-CoV-2. Cabe aqui salientar que, para que haja uma manutenção sustentada do controle dos índices após o período de lockdown, se faz necessária, além de uma campanha de vacinação em massa, a manutenção das medidas sanitárias básicas conforme recomenda a Organização Mundial da Saúde.

\section{Agradecimentos}

Os autores agradecem o apoio financeiro da FAPESP (Fundação de Amparo à Pesquisa do Estado de São Paulo - Processo 2013/07375-0: CEPID-CeMEAI).

\section{Referências}

[1] Amaral, F. et al. Towards Providing Effective Data-Driven Responses to Predict the Covid-19 in São Paulo and Brazil. Sensors, volume 21, 2021.

[2] Bedford, J. et al. COVID-19: towards controlling of a pandemic. The lancet, volume 395, n.10229, pages 1015-1018, 2020.

[3] Bi, Q. et al. Epidemiology and transmission of COVID-19 in 391 cases and 1286 of their close contacts in Shenzhen, China: a retrospective cohort study. The Lancet Infectious Diseases, volume 20, n.8, pages 911-919, 2020.

[4] Bo, Y. et al. Effectiveness of non-pharmaceutical interventions on COVID-19 transmission in 190 countries from 23 January to 13 April 2020. International Journal of Infectious Diseases, volume 102, pages 247-253, 2021.

[5] Davies, N. G. et al. Effects of non-pharmaceutical interventions on COVID-19 cases, deaths, and demand for hospital services in the UK: a modelling study. The Lancet Public Health, volume 5, n.7, pages e375-e385, 2020.

[6] Ortega, F. and Orsini, M. Governing COVID-19 without government in Brazil: Ignorance, neoliberal authoritarianism, and the collapse of public health leadership, Global Public Health, volume 15, n.9, pages 1257-1277, 2020.

[7] Phillips, B. et al. Coronavirus 10-day forecast Project. Faculty of Science - Universidade de Melbourne, www.covid19forecast.science.unimelb.edu.au/. Acessado em: 14 de abril de 2021. 